\title{
Cosmic superconductivity
}

( o superconducting phases appear in nature? Given that many metals are superconductors at very low temperatures, it's tempting to take for granted the notion that this fundamentally quantum property is a 'part of nature.' But of course most metals rarely feature in the natural world in their elemental state, and even when they do it is far from clear that their purity would be sufficient to support superconducting phases. Lead, with a superconducting transition temperature of $7.2 \mathrm{~K}$, sometimes occurs in its native state, but superconductivity in natural samples has never been reported. In fact, until now the only previous claim of superconductivity in a natural material has been in the rare layered copper sulfide mineral covellite ${ }^{1}$, which has a transition temperature of about $1.6 \mathrm{~K}$.

Now Wampler et al. describe evidence of superconductivity in phases within two meteorites ${ }^{2}$. They used a technique called magnetic field modulated microwave spectroscopy (MFMMS), an ultrasensitive method that identifies a superconducting transition as a peak in the derivative of microwave absorption with applied magnetic field, as the temperature is changed ${ }^{3}$. One sample was an iron meteorite found in Mundrabilla, Australia, the first fragments of which were identified (by happy coincidence) in 1911, when Heike Kamerlingh Onnes discovered superconductivity. The other was one of a group of meteorites also found in Australia and first described in 1998; it is a stony, carbon-rich meteorite called a ureilite. Both showed MFMMS peaks at around 5-6 K.

To try to characterize the phases responsible for this behaviour, Wampler et al. narrowed down the sample materials grain by grain to find those with the largest superconducting fraction. These proved also to have a metallic lustre, suggesting that the two properties are connected. Energy-dispersive $\mathrm{X}$-ray spectroscopy, which can reveal the chemical elements present, showed significant amounts of lead, tin and indium in the Mundrabilla material, and of just the latter two elements in the 1998 meteorite. So the superconductivity seems to stem from alloys of these elements.

It is all very curious - but so what? Is this just an odd quirk of nature? Perhaps not - for unlike terrestrial covellite, the parent bodies of meteorites may actually find themselves in conditions where the superconductivity is active. Some regions of the interstellar medium may have temperatures of $5 \mathrm{~K}$ or lower, although star-forming dense molecular clouds are typically several degrees warmer. Such superconducting particles in space might support microscopic currents if they experience transient magnetic fields, and these in turn would generate magnetic fields of their own. Molecular clouds do possess magnetic fields, although their origin is not well understood ${ }^{4}$ - and these could play a role in how dust grains aggregate into protostellar bodies. Of course, there are key questions about how abundant superconducting

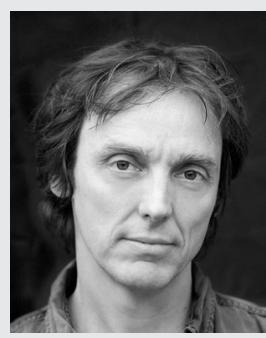

Philip Ball

grains would be anyway, but it is at least conceivable that superconductivity influences the origins of stars and planets.

Perhaps, though, the implications are broader. There has long been discussion of whether fundamentally quantum effects, such as tunnelling ${ }^{5}$ or quantum coherence ${ }^{6}$, might play a role in the natural world, influencing (respectively) the origins of homochirality or the operation of photosynthesis. We might regard these new findings too as a hint that nature does not work solely in the classical limit.

Published online: 24 April 2020 https://doi.org/10.1038/s41563-020-0671-2

References

1. Di Benedetto, F. et al. Eur. J. Mineral. 18, 283-287 (2006).

2. Wampler, J., Thiemens, M., Zhu, Y. \& Schuller, I. K. Proc. Natl Acad. Sci. USA https://doi.org/10.1073/ nas. 1918056117 (2020)

3. Ramírez, J. G., Basaran, A. C., de la Venta, J., Pereiro, J. \& Schuller, I. K. Rep. Progr. Phys. 77, 093902 (2014).

4. Crutcher, R. M. Annu. Rev. Astr. Astrophys. 50, 29-63 (2012).

5. Goldanskii, V. I. Sci. Am. 254(February), 46-53 (1986).

6. Engel, G. S. Procedia Chem. 3, 222-231 (2011). 